https://helda.helsinki.fi

Ceramides : A Cause of Insulin Resistance in Nonalcoholic Fatty Liver Disease in Both Murine Models and Humans

\title{
Yki-Järvinen, Hannele
}

2020-04

Yki-Järvinen, H 2020 , ' Ceramides : A Cause of Insulin Resistance in Nonalcoholic Fatty

Liver Disease in Both Murine Models and Humans ', Hepatology , vol. 71 , no. 4 , pp.

1499-1501 . https://doi.org/10.1002/hep.31095

http://hdl.handle.net/10138/328477

https://doi.org/10.1002/hep.31095

acceptedVersion

Downloaded from Helda, University of Helsinki institutional repository.

This is an electronic reprint of the original article.

This reprint may differ from the original in pagination and typographic detail.

Please cite the original version. 
Article type : Hepatology Elsewhere

\section{Ceramides - a cause of insulin resistance in NAFLD in both murine models and humans}

Hannele Yki-Järvinen ${ }^{1,2}$, M.D., F.R.C.P.

${ }^{1}$ University of Helsinki and Helsinki University Hospital, Department of Medicine and ${ }^{2}$ Minerva Foundation Institute for Medical Research, Helsinki, Finland

\section{Abbreviations:}

NAFLD, nonalcoholic fatty liver disease; CV, cardiovascular disease; PNPLA3, patatin-like phospholipase-domain containing protein 3; $\mathrm{NASH}$, non-alcoholic steatohepatitis; $\mathrm{C}_{16: 0}$, palmitate; palmitoyl-CoA, palmitoyl coenzyme $A_{\text {; }}$ CerS, ceramide synthase; $C_{16: 0}$-ceramides, ceramides with a fatty acyl chain containing 16 carbons and 0 double bonds; DES, the enzyme dihydroceramide desaturase; degs1, the dihydroceramide desaturase 1 gene; ob/ob mouse, a leptin-deficient obese mouse; srebf1, sterol regulatory element transcription factor 1 ; CD36, fatty acid translocase; DNL, de novo lipogenesis

Potential conflicts of interest: Dr. Yki-Järvinen consults for Novo Nordisk, MSD, Lilly and Gilead.

Address for correspondence:

Hannele Yki-Järvinen, M.D., F.R.C.P., Haartmaninkatu 8, FIN - 00290 Helsinki, Finland

E-mail: Hannele.Yki-Jarvinen@helsinki.fi. Tel: +358 504271664

WORD COUNT OF TEXT AND FIGURE LEGEND INCLUDING REFERENCES 1516

This article has been accepted for publication and undergone full peer review but has not been through the copyediting, typesetting, pagination and proofreading process, which may lead to differences between this version and the Version of Record. Please cite this article as doi: 10.1002/hep.31095

This article is protected by copyright. All rights reserved 
Patients with both NAFLD and the insulin resistance/metabolic syndrome ('Metabolic NAFLD') have an increased risk of developing type 2 diabetes and CVD. Triglycerides, contained within hepatic lipid droplets, themselves are, however, inert and not sufficient to cause insulin resistance. In numerous murine models, the liver accumulates triglycerides without becoming insulin resistant. Patients with NAFLD due to genetic variants such as the I148M variant in PNPLA3 ('PNPLA3 NAFLD'), are not insulin resistant and their risk of CVD is neutral (1). Nevertheless, carriers of this gene variant have a 2-3-fold increased risk of developing nonalcoholic steatohepatitis (NASH) and advanced fibrosis. There is thus a dissociation between steatosis and insulin resistance also in human NAFLD. What might then be the reason why steatosis is accompanied by insulin resistance in 'Metabolic NAFLD?

Upon a closer look, not all fat is alike, even in the human liver. In 'Metabolic NAFLD', triglycerides are predominantly saturated but polyunsaturated in 'PNPLA3 NAFLD' (2). In 'Metabolic NAFLD' but not 'PNPLA3 NAFLD', the liver is enriched with markers of the pathway of de novo ceramide synthesis. Overeating saturated fat saturates the human liver triglyceride composition (3). In addition, although synthesis of liver triglycerides is not the primary pathway to utilize carbohydrate, their excess consumption stimulates DNL and produces exclusively saturated fatty acids (Fig. 1). This is important as the NAFLD epidemic has paralleled intake of simple sugars, not fat (4). Saturated fatty acids, especially palmitate $\left(C_{16: 0}\right)$ stimulate formation of ceramides via de novo ceramide synthesis (Fig. 1). Ceramides are biologically active sphingolipids that impair insulin action, and induce inflammation and mitochondrial dysfunction (5).

In the de novo pathway of ceramide synthesis, palmitate-derived palmitoylcoenzyme A reacts with serine to produce a sphingoid backbone that subsequently incorporates additional fatty acids via the action of six fatty acid specific ceramide synthetase (CerS) enzymes. Of these, CerS5 and CerS6 add saturated fatty acid $\mathrm{C}_{16: 0}$ to the sphingoid backbone. Liver-specific CerS6 but not CerS5 knock-out mice are protected against saturated fatty acid-induce insulin resistance and steatosis $(6,7)$. This difference was recently attributed to the ability of CerS6 derived sphingolipids to bind mitochondrial fission factors and promote fatty acid induced mitochondrial fragmentation on subcellular pools of $\mathrm{C}_{16: 0}$ ceramides (7).

The final step of ceramide formation involves insertion of a single double bond to dihydroceramide by the enzyme DEGS1. DEGS1 is the isoform resident in virtually all cells. 
Recently, Chaurasia et al examined the importance of ceramides as compared to dihydroceramides in inducing steatosis and insulin resistance (8). Degs1 was conditionally deleted in all tissues of adult mice and specifically from liver and/or adipose tissue. Although germline deletion of Degs1 is lethal, ablation of the DEGS1 enzyme from adult animals produced no side effects. Excising Degs1 from intestinal or myeloid cells had no effect on any metabolic parameters (8). Liver-targeted Degs1 in leptin-deficient ob/ob mice decreased ceramide/ dihydroceramide ratios in serum, liver and muscle, while decreases in these ratios were observed in adipose tissues and muscle after adipose tissue Degs1 removal. Deletion of Degs 1 from the liver or adipose tissue or both improved insulin sensitivity of glucose metabolism and reduced liver steatosis without affecting body weight, food intake, ambulatory activity or oxygen consumption in mice fed a high fat ( $60 \%$ fat, $20 \%$ carbohydrate) diet. The effect of liver-targeted deletion on liver triglycerides was more robust than that in adipose tissue. Degs 1 inhibition in the liver markedly down-regulated the master regulator of triglyceride and sterol production, Srebf1 and its downstream targets. In adipocytes, ceramides but not dihydroceramides blocked release of fatty acids from adipocytes by inhibiting hormone-sensitive lipase. Ceramides also increased insulin-stimulated uptake of free fatty acids by primary hepatocytes (Fig. 1). Thus, ceramides promoted storage of fatty acids as triglycerides and prevented peripheral fatty acid release. The authors surmised that ceramides serve to protect against of toxic effects of free fatty acids under conditions where energetic needs of the tissue have been met (8). What a difference a single double bond can make!

The series of studies manipulating different steps of de novo ceramide synthesis in mice make up a consistent story (Fig. 1). Inhibition of ceramide synthesis from saturated fatty acids at multiple enzymatic steps improve insulin sensitivity of glucose metabolism and hepatic triglyceride storage. Furthermore, earlier work showed targeted induction of ceramide degradation via stimulation of acid ceramidase in the liver and adipose tissues to have similar effects (9). Adiponectin, the best characterized insulin sensitizing adipokine in murine models and humans, shares these beneficial metabolic effects and acts by lowering of hepatic ceramide content via stimulation of ceramidase activity.

The intervention data obtained by studying genetically engineered murine models and human cross-sectional data suggest ceramides are strongly implicated in the pathogenesis of 
NAFLD. These data are of particular interest in the face of several recent large prospective studies evaluating serum sphingolipids as cardiometabolic biomarkers (5). Especially ceramides with saturated fatty acyl side chains predict risks of insulin resistance, diabetes and CVD (5). Ceramides appear to be prognostic for CVD, the main cause of death in patients with NAFLD and $\mathrm{NASH}$, even independent of LDL cholesterol and other classic risk factors. At present there are no data to suggest that measurement circulating ceramides would help the hepatologist to assess future risk of diabetes or CVD in a patient with NAFLD beyond routinely available clinical data. The presence of features of the metabolic syndrome is enough to suggest the patient has hepatic insulin resistance. The pathogenesis of NASH and fibrosis are much more complicated than just 'insulin resistance'. Perhaps the best proof of this is that common genetic variants are associated with NASH but not with ceramide accumulation or insulin resistance (2).

Time will tell whether inhibition of the de novo ceramide synthetic pathway will become as metabolically beneficial in humans as it is mice. Many problems have been solved in mice but successes have not been replicated in humans. Mice and men are separated by $65-80$ million years of evolution. Obese humans have hyperleptinemia and are not leptin-deficient. The average American diet contains $35 \%$, not $60 \%$ fat. Does it make sense to block pathways such as DNL or ceramide synthesis which help to channel excess sugars and fatty acids to triglycerides under conditions of energy surplus? Wouldn't it be wiser to simply reduce the intake of these nutrients? The NAFLD epidemic shows that this inexpensive treatment that should underpin the management is not utilized. There is therefore a need to discover new pharmacotherapies. Perhaps it will be easier to block de novo ceramide synthesis in human beings than it is to change their behavior.

This article is protected by copyright. All rights reserved 
Fig. 1. A schematic overview of the de novo ceramide synthetic pathway. Excess intake saturated fat increases the content of saturated fatty acids (SFAs) such as palmitate $\left(C_{16: 0}\right)$ in the human liver and induces insulin resistance (3). Overeating simple sugars stimulates de novo lipogenesis (DNL) which also produces saturated fatty acids. Under conditions of energy surplus, palmitoylcoenzyme $A(C O A)$ enters the de novo ceramide synthetic pathway which produces dihydroceramides. The enzyme dihydroceramide desaturase 1 (DEGS1) adds a single double bond to dihydroceramides thereby converting them to ceramides. Ceramides stimulate storage of fatty acids (FAs) in liver triglycerides (TGs) via stimulation of expressions of sterol regulatory element binding transcription factor 1 (srebf1) in hepatocytes, and hormone sensitive lipase (hsl) in adipocytes. The red cross denotes consequences of deletion of DEGS1 from the liver (8). This includes increases in dihydroceramides and decreases in hepatic and circulating ceramides, decreased uptake of free fatty acids (FAs), lowering of liver TGs and enhanced insulin sensitivity of hepatic glucose production (HGP). Other abbreviations: CM=chylomicrons, VLDL=very lowdensity lipoprotein, $S=$ serum. 


\section{REFERENCES}

1.

Liu DJ, Peloso GM, Yu H, Butterworth AS, Wang X, Mahajan A, Saleheen D, et al. Exome-wide association study of plasma lipids in $>300,000$ individuals. Nat Genet 2017;49:17581766.

2.

Luukkonen PK, Zhou Y, Sadevirta S, Leivonen M, Arola J, Oresic M, Hyotylainen T, et al. Hepatic ceramides dissociate steatosis and insulin resistance in patients with non-alcoholic fatty liver disease. J Hepatol 2016;64:1167-1175.

3. Luukkonen PK, Sadevirta S, Zhou Y, Kayser B, Ali A, Ahonen L, Lallukka S, et al. Saturated Fat Is More Metabolically Harmful for the Human Liver Than Unsaturated Fat or Simple Sugars. Diabetes Care 2018;41:1732-1739.

4. Writing Group M, Lloyd-Jones D, Adams RJ, Brown TM, Carnethon M, Dai S, De Simone G, et al. Heart disease and stroke statistics--2010 update: a report from the American Heart Association. Circulation 2010;121:e46-e215.

5. Holland WL, Summers SA. Strong Heart, Low Ceramides. Diabetes 2018;67:14571460.

6. Hla T, Kolesnick R. C16:0-ceramide signals insulin resistance. Cell Metab 2014;20:703-705.

7. Hammerschmidt P, Ostkotte D, Nolte H, Gerl MJ, Jais A, Brunner HL, Sprenger HG, et al. CerS6-Derived Sphingolipids Interact with Mff and Promote Mitochondrial Fragmentation in Obesity. Cell 2019;177:1536-1552 e1523.

8. Chaurasia B, Tippetts TS, Mayoral Monibas R, Liu J, Li Y, Wang L, Wilkerson JL, et al. Targeting a ceramide double bond improves insulin resistance and hepatic steatosis. Science 2019;365:386-392.

9. Xia JY, Holland WL, Kusminski CM, Sun K, Sharma AX, Pearson MJ, Sifuentes AJ, et al. Targeted Induction of Ceramide Degradation Leads to Improved Systemic Metabolism and Reduced Hepatic Steatosis. Cell Metab 2015;22:266-278.

This article is protected by copyright. All rights reserved 


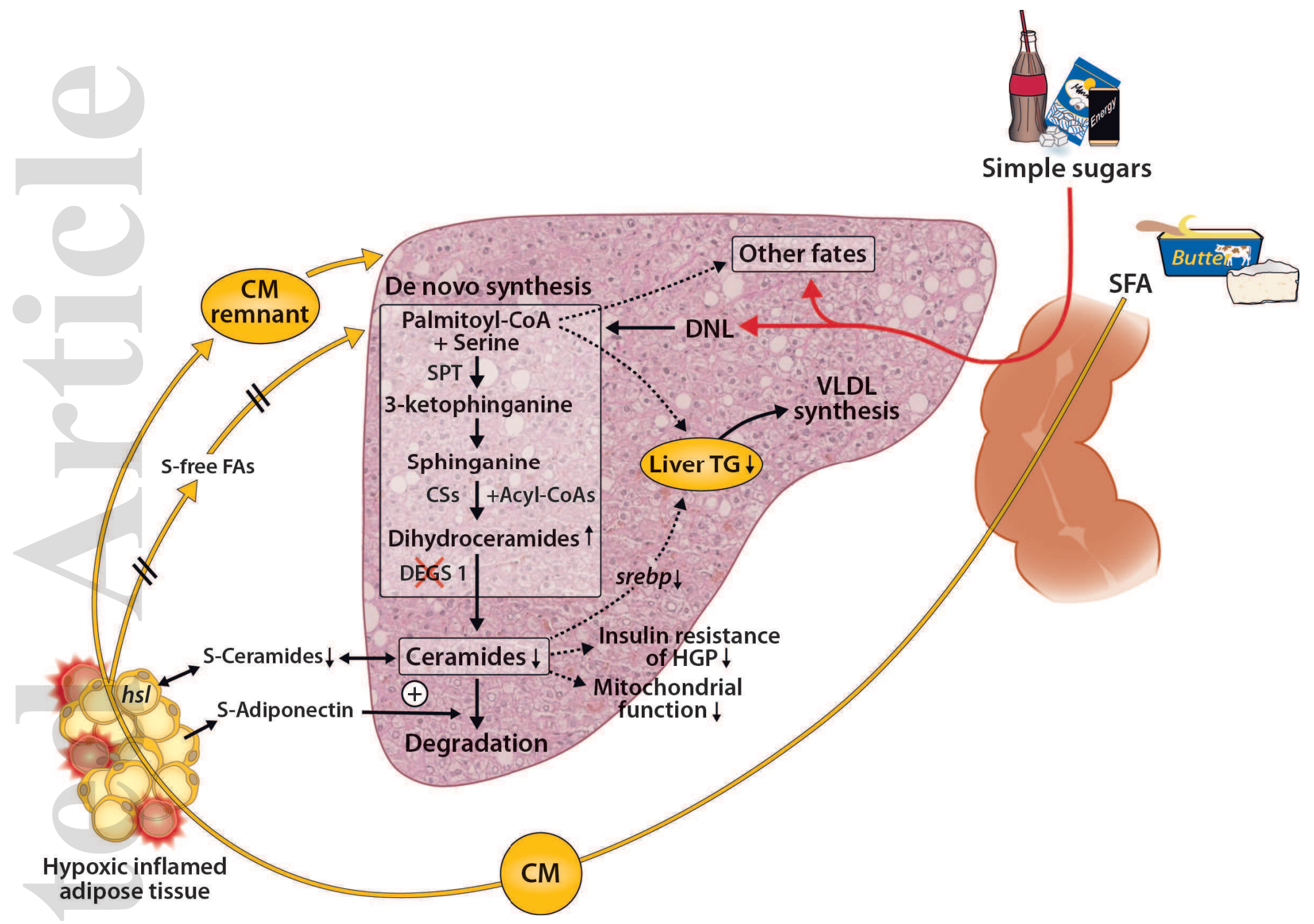

hep_31095_f1.eps

This article is protected by copyright. All rights reserved 